\title{
Arteriogenesis - Therapeutic Stimulation of the Coronary Collateral Circulation
}

\section{Pascal Meier*}

General and Interventional Cardiology, University College London Hospitals, London, UK

\section{Clinical Relevance}

The coronary arteries have been regarded as end-arteries for a long time. Functionally, they are end-arteries in most cases indeed. However, there are interconnecting collateral channels between the large epicardial arteries which are sufficient to prevent myocardial ischemia during an acute vessel occlusion in about $1 / 4$ to $1 / 3$ of patients [1]. There are even very impressive rare examples of patients with left main artery occlusions who never had a myocardial infarction and who have very mild symptoms [2]. Despite such anecdotes which clearly illustrate the importance of the collateral circulation in human in some extreme situations, the overall relevance of collaterals is still not very clear. Even a very recent large study failed to show a clear clinical benefit of collaterals [3]. However, this study had some limitations and pooling the results of all currently available data on this topic clearly shows an association between the degree of collateralization and survival [4]. This meta-analysis included a total of 12 studies enrolling 6529 patients. Patients with a well developed collateral network had a $36 \%$ reduced mortality risk. One potential mechanism is the protective effect of the collateral circulation on the repolarization during an acute occlusion of a main coronary artery which usually puts the patient at risk for dangerous ventricular arrhythmias [5].

On the other hand, collaterals are clearly associated with an increased risk for restenosis after a coronary revascularization [6]. It is not clear whether this association is causal or whether they are simply a marker of more advanced and aggressive disease.

Regardless, the coronary collateral circulation seems to have a major clinical impact and unfortunately, only a minority of patients have sufficient collaterals to maintain sufficient myocardial perfusion during an abrupt occlusion of a coronary artery.

\section{Therapeutic Implications}

The key question is whether we can improve the coronary collateral circulation in human. Multiple studies in animals have demonstrated that collaterals can be improved with therapeutics. However, the translation to clinical application has failed in most cases. However, a few proofof-concept studies have shown promising results, for example with the glycoprotein granulocyte colony stimulating factor G-CSF [7].

It is important to understand the mechanism of collateral growth. The mechanism is called arteriogenesis which differs from angiogenesis. The letter means a sprouting of de-novo capillaries while arteriogenesis describes a process of remodeling of pre-existing interconnecting arteries. The trigger for the two processes differs, while angiogenesis is induced by hypoxia, arteriogenesis is induced by fluid shear stress on the endothelial cells. In arteriogenesis, monocytes play a pivotal role. The main role is probably paracrine; they release chemokines, growth factors and proteases which are involved in vascular growth.

Therefore, new therapies need to consider these mechanisms. Monocytes are potential targets, as illustrated by effect of G-CSF [7]. Another option is to influence the shear stress on endothelial cells. This has actually been tested successfully in a first small trial. Shear stress was increased via external counter pulsation. The collateral function significantly improved with this treatment [8].

The therapeutic stimulation of the human collateral circulation is still at an early stage but hopefully, ongoing and future research will hopefully help to improve our understanding of arteriogenesis and to specifically target this process therapeutically in order to improve patients outcomes [9].

\section{References}

1. Seiler C (2010) The human coronary collateral circulation. Eur J Clin Invest 40 465-476.

2. Meier $P$ (2011) The sword of Damocles: an illustrative example of the life-saving effect of the collateral circulation. J Invasive Cardiol 23: E47-E48.

3. Steg PG, Kerner A, Mancini GB, Reynolds HR, Carvalho AC et al. (2010) Impact of collateral flow to the occluded infarct-related artery on clinical outcomes in patients with recent myocardial infarction: a report from the randomized occluded artery trial. Circulation 121: 2724-2730.

4. Meier P, Hemingway H, Lansky AJ, Knapp G, Pitt B, et al. (2012) The impact of the coronary collateral circulation on mortality: a meta-analysis. Eur Heart J 33 : 614-621.

5. Meier P, Gloekler S, de Marchi SF, Zbinden R, Delakretaz E, et al. (2010) An indicator of sudden cardiac death during brief coronary occlusion: electrocardiogram QT time and the role of collaterals. Eur Heart J 31: 1197-1204.

6. Meier P, Indermuehle A, Pitt B, Traupe T, de Marchi SF, et al. (2012) Coronary collaterals and risk for restenosis after percutaneous coronary interventions: a meta-analysis. BMC Med 10: 62.

7. Meier P, Gloekler S, de Marchi SF, Indermuehle A, Rutz T, et al. (2009) Myocardial salvage through coronary collateral growth by granulocyte colonystimulating factor in chronic coronary artery disease: a controlled randomized trial. Circulation 120: 1355-1363.

8. Gloekler S, Meier P, de Marchi SF, Rutz T, Traupe T, et al. (2010) Coronary collateral growth by external counterpulsation: a randomised controlled trial Heart 96: 202-207.

9. Schaper W (2012) Collateral vessels reduce mortality. Eur Heart J 33: 564-566.

${ }^{*}$ Corresponding author: Pascal Meier, General and Interventional Cardiology, University College London Hospitals, London, UK, E-mail: pascalmeier74@gmail.com

Received October 22, 2012; Accepted October 23, 2012; Published October 25 2012

Citation: Meier P (2012) Arteriogenesis - Therapeutic Stimulation of the Coronary Collateral Circulation. Cardiol Pharmacol 1:e101. doi:10.4172/2329-6607.1000e101

Copyright: () 2012 Meier P. This is an open-access article distributed under the terms of the Creative Commons Attribution License, which permits unrestricted use, distribution, and reproduction in any medium, provided the original author and source are credited. 